\title{
Relación entre edad cronológica y estadios de mineralización del tercer molar inferior en radiografías panorámicas digitales.
}

\section{Relationship between chronological age and mineralization stages of the lower third molar on digital panoramic radiographs.}

\author{
Andrés Domínguez-Quinteros ${ }^{1}$, Magdalena Molina-Barahona ${ }^{2 *}$, Ana Cristina Vásquez-Palacios ${ }^{3}$, Liliana \\ Encalada-Verdugo ${ }^{4}$, Stephany Paladines-Calle ${ }^{4}$ \\ ${ }^{1}$ Especialista en Radiología Bucal y Maxilofacial, \\ 2 Docente de Radiología Bucal y Maxilofacial, carrera de Odontología, Universidad Católica de Cuenca-Ecuador. \\ ${ }^{3}$ Docente de Periodoncia, carrera de Odontología, Universidad Católica de Cuenca-Ecuador. \\ ${ }^{4}$ Máster en Estomatología, docente de la carrera de Odontología, Universidad Católica de Cuenca-Ecuador. \\ 5 Odontóloga de práctica privada. \\ *dramolinabarahona@gmail.com
}

\begin{abstract}
Resumen
Objetivo: Relacionar la edad cronológica y la mineralización del tercer molar inferior en radiografías panorámicas digitales de pacientes entre 7 a 23 años que acudieron a un centro radiológico privado.Materiales y métodos:Se realizó un estudio descriptivo, retrospectivo, en el cual se analizó un total de 596 radiografías panorámicas, tomadas durante el período 20162017, posteriormente se realizó la comparación estadística de los estadios de mineralización según el sexo a través de la prueba T student y U de Mann-Witney, asimismo se determinó el coeficiente de correlación Rho de Spearman entre la edad cronológica y estadio de mineralización.Resultados: La mayor proporción de radiografías evaluadas fueron del sexo femenino. El promedio de edad en la pieza 38 para el sexo femenino comienza a los 9,15 años y termina a los 21,19 años, en el sexo masculino inicia a los 10,00 años y termina a los 21,02 años. El promedio de edad en la pieza 48 para el sexo femenino comienza a los 9,50 años y termina a los 21,21 años, en el sexo masculino comienza a los 9,65 años y termina a los 21,01 años. Conclusiones: Se corroboro una muy alta correlación entre la edad cronológica y los estadios de mineralización del tercer molar inferior en radiografías panorámicas digitales.
\end{abstract}

Palabras clave: Tercer Molar, Determinación de la Edad por los Dientes, Radiografía Panorámica.

Objective: Relate the chronological age and mineralization of the lower third molar in digital panoramic radiographs of patients between 7 and 23 years old who went to a private radiological center. Materials and methods: A descriptive, retrospective study was carried out, in which a total of 596 panoramic radiographs were analyzed, taken during the 20162017 period, the statistical comparison of the stages of mineralization according to sex was subsequently made through the Student T test and Mann-Witney U test, recently Spearman's Rho correlation coefficient between chronological age and mineralization stage was determined. Results: The highest proportion of radiographs evaluated were female. The average age in piece 38 for the female sex begins at 9.15 years and ends at 21.19 years, in the male sex it begins at 10.00 years and ends at 21.02 years. The average age in piece 48 for the female sex begins at 9.50 years and ends at 21.21 years, in the male sex it begins at 9.65 years and ends at 21.01 years. Conclusions: A very high correlation between chronological age and the mineralization stages of the lower third molar was confirmed in digital panoramic radiographs.

Key words: Third Molar, Determination of Age by Teeth, Panoramic Radiography.

\section{INTRODUCCIÓN}

En el aspecto jurídico legal la Odontología Forense ha ganado notable importancia respecto a la estimación de la edad, mediante el análisis del proceso de madurez dental. Entre los métodos convencionales se encuentra la maduración morfológica (talla y peso), la evaluación clínica- radiográfica de la edad dental y la madurez esquelética obtenida mediante la radiografía carpal, que proporciona información hasta los 16 años de edad. ${ }^{1}$

Uno de los métodos más empleados en la evaluación clínica-radiográfica de la edad dental es el método de De- 
mirjian, ha sido ampliamente utilizado alrededor del mundo, donde se han encontrado variaciones durante su aplicación. Incluye 8 estadios, a partir del comienzo de la formación de la corona, terminando con el cierre del ápice radicular; cada estadio presenta características muy específicas y claras que diferencian uno de otro. ${ }^{2}$

Descripción de los estadios de formación.

- Estadio A: Iniciación de la mineralización de la superficie oclusal con presencia de puntos de calcificación sin unión entre estos.

- Estadio B: Fusión de dos o más puntos de mineralización lográndose observar el contorno oclusal.

- Estadio C: Esmalte cien por ciento mineralizado con el inició del depósito de dentina.

- Estadio D: Formación coronal completa hasta el límite amelocementario con el inicio de la formación radicular.

- Estadio E: Corta formación radicular, comienza la bifurcación entre las raíces y las longitudes radiculares son menores a las de la corona.

- Estadio F: Longitud de la raíz respecto a la corona es igual o mayor. Las terminaciones radiculares presentan un aspecto en forma de embudo.

- Estadio G: Formación radicular casi completa, ápices parcialmente abiertos y el espacio de ligamento periodontal a nivel apical se observa ensanchado.

- Estadio H: Formación radicular culminada, con membrana periodontal uniforme. ${ }^{3,4}$

Los terceros molares son las únicas piezas dentales que no completan la formación total de su raíz hasta los 25 años de edad. La correlación entre las etapas de mineralización y la edad cronológica, nos aproximan a determinar la transición entre el estado juvenil y el de adulto mayor de 18 años. ${ }^{5,6}$

En el año 2005 Olze y colaboradores realizaron una investigación en la cual evaluaron el estadio de mineralización de los terceros molares inferiores utilizando la clasificación de Gleiser y Hunt, Demirjian, Gustafson y Koch, Harris y Nortje y Kullman. Los autores llegaron a la conclusión que el método de Demirjian es uno de los métodos más efectivos para evaluar la mineralización de los terceros molares y estimar la edad en el área forense. ${ }^{7}$

El propósito de la presente investigación fue establecer la relación entre la edad cronológica y las etapas de calcificación de los terceros molares inferiores mediante radiografías panorámicas, de pacientes entre 7 a 23 años, AzoguesEcuador.

\section{MATERIALES Y MÉTODOS}

Se realizó un estudio de tipo descriptivo, transversal, observacional y analítico. La selección de las radiografías panorámicas se realizó a través de muestreo por conveniencia, utilizando la totalidad de radiografías que cumplían con los criterios de selección. Teniendo una muestra de 596 radiografías panorámicas digitales, las cuales fueron tomadas en un centro radiológico privado de la Ciudad de Azogues - Ecuador, durante el periodo enero 2016 - diciembre 2017.

Se incluyeron radiografías de pacientes entre 7 a 23 años de edad que presentaban ambos terceros molares inferiores. Se excluyeron radiografías panorámicas que presentaban patologías tumorales o quísticas, terceros molares con anomalías dentarias y radiografías de mala calidad diagnóstica.

El investigador principal fue capacitado y calibrado por un Radiólogo Oral y Maxilofacial. Dicha calibración fue desarrollada en radiografías panorámicas digitales tomadas con el equipo radiográfico Veraviewepocs (J. MORITAMFJ.CORP/ MODEL: X550CP-DC-UL, US). Los estadios encontrados se compararon con los del experto (interobservador) y con los del investigador (intraobservador), el valor de kappa obtenido fue de 0,975 y 0,95 respectivamente. De forma similar se recolectaron los datos de sexo, edad cronológica y estadio de Demirjian de la pieza 3.8 y 4.8. Se realizó la comparación de los estadios de mineralización entre las piezas con la finalidad de verificar si existe diferencias.

Para el análisis estadístico de los datos se utilizó el paquete estadístico STATA versión 12. Donde se efectuó la comparación estadística de los estadios de mineralización según el sexo a través de la prueba $\mathrm{T}$ student para aquellos grupos que presentaron una distribución normal, y la prueba U de Mann-Witney para aquellos grupos que presentaron una distribución anormal, con la finalidad de verificar si existe diferencia entre el sexo masculino y femenino. Asimismo, se determinó el coeficiente de correlación Rho de Spearman entre la edad cronológica y estadio de mineralización según sexo.

\section{RESULTADOS}

De las 596 radiografías, 349 (58,6\%) fueron pacientes de sexo femenino y $247(41,4 \%)$ del sexo masculino. En el género femenino la mayor proporción de pacientes tuvo una edad entre 22,00 y 22,99 años; mientras que el sexo masculino existió un mayor número de radiografías de paciente con edades entre 17,00 y 17,99 .

Al evaluar la pieza 3.8 (Tabla 1), se encontró que el promedio de edad cronológica en pacientes del sexo femenino en el estadio Demirjian D fue de 13,25 $\pm 2,17$ y en el estadio $H$ fue de 21,19 $\pm 2,13$ años. En pacientes del sexo 
masculino el estadio D fue de 13,07 $\pm 2,09$ y el estadio $\mathrm{H}$ $21,02 \pm 1,54$ años. Corroborándose que no se encontraron diferencias estadísticamente significativas, según sexo en los estadios de mineralización. Y con respecto a la relación entre la edad cronológica y la mineralización del tercer molar tanto derecho como izquierdo, se presentó una correlación muy alta para ambos sexos. Asimismo, se constataron resultados similares en la pieza 4.8 (Tabla 2).

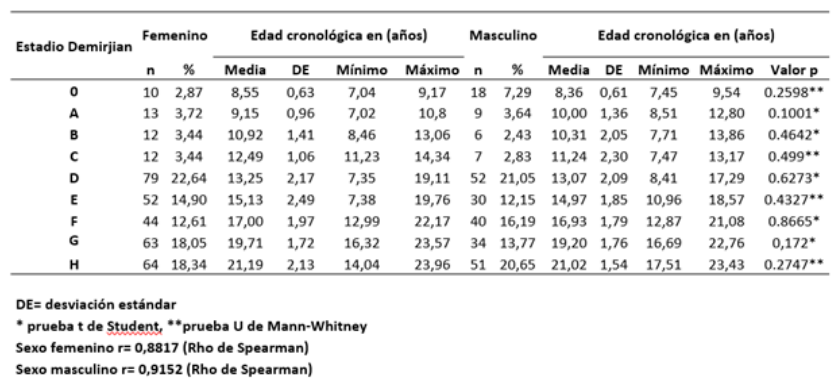

Tabla 1. Distribución de estadios de Demirjian en la pieza 3.8 según sexo y edad cronológica.

\begin{tabular}{ccccccccccc}
\hline $\begin{array}{c}\text { Estadio de } \\
\begin{array}{c}\text { Mineralización } \\
\text { en pieza 48 }\end{array}\end{array}$ & O & A & B & C & D & E & F & G & H & Total \\
\cline { 2 - 10 } & Estadio de Mineralización en Pieza 38 \\
\hline 0 & 28 & 3 & 2 & - & - & - & - & - & - & 34 \\
A & - & 18 & 2 & - & 1 & - & - & - & - & 21 \\
B & - & 1 & 14 & - & - & - & - & - & - & 15 \\
C & - & - & - & 15 & 2 & - & - & - & - & 17 \\
D & - & - & - & 4 & 122 & 7 & 1 & - & - & 134 \\
E & - & - & - & - & 5 & 71 & 4 & 1 & - & 80 \\
F & - & - & - & - & 1 & 4 & 78 & 13 & 1 & 97 \\
G & - & - & - & - & - & - & 1 & 77 & 6 & 84 \\
H & - & - & - & - & - & - & - & 6 & 108 & 114 \\
Total & 28 & 22 & 18 & 19 & 131 & 82 & 84 & 97 & 115 & 596 \\
\hline
\end{tabular}

*Porcentaje de acuerdo de $88,92 \%$

Tabla 3. Comparación de estadios de mineralización según Demirjian entre las piezas 38 y 48 .

A la evaluación estadística de la probabilidad de tener 18 años o más según el estadio, en la pieza 3.8 en el sexo femenino, el estadio que determina es el $\mathrm{H}$ en $93,8 \%$ y en el sexo masculino de igual manera es el $\mathrm{H}$ en un $96,1 \%$. Similares resultados se encontraron en la pieza 4.8, para el sexo femenino el estadio $\mathrm{H}$ estuvo presente en el $96,1 \%$ de la población y en el sexo masculino en el $96 \%$ (Tabla 4).

\begin{tabular}{ccccc}
\hline $\begin{array}{c}\text { Estadio de } \\
\text { Demirjian }\end{array}$ & \multicolumn{2}{c}{ Pieza 38 } & \multicolumn{2}{c}{ Pieza 48 } \\
\cline { 2 - 5 } & $\begin{array}{c}\text { Menor de 18 } \\
\text { años }\end{array}$ & $\begin{array}{c}\mathbf{1 8} \text { o más de } \mathbf{1 8} \\
\text { años }\end{array}$ & $\begin{array}{c}\text { Menor de 18 } \\
\text { años }\end{array}$ & $\begin{array}{c}\mathbf{1 8} \text { o más de 18 } \\
\text { años }\end{array}$ \\
\hline Femenino & & & & \\
Estadio D & $97,4 \%$ & $2,6 \%$ & $100,0 \%$ & $0,0 \%$ \\
Estadio E & $94,0 \%$ & $6,0 \%$ & $90,4 \%$ & $9,6 \%$ \\
Estadio F & $74,4 \%$ & $25,6 \%$ & $66,7 \%$ & $33,3 \%$ \\
Estadio G & $16,1 \%$ & $83,9 \%$ & $11,3 \%$ & $88,7 \%$ \\
Estadio H & $6,3 \%$ & $93,8 \%$ & $6,3 \%$ & $93,8 \%$ \\
Masculino & & & & \\
Estadio D & $100,0 \%$ & $0,0 \%$ & $100,0 \%$ & $0,0 \%$ \\
Estadio E & $96,7 \%$ & $3,3 \%$ & $100,0 \%$ & $0,0 \%$ \\
Estadio F & $75,0 \%$ & $25,0 \%$ & $74,4 \%$ & $25,6 \%$ \\
Estadio G & $29,4 \%$ & $70,6 \%$ & $19,4 \%$ & $80,6 \%$ \\
Estadio H & $3,9 \%$ & $96,1 \%$ & $4,0 \%$ & $96,0 \%$ \\
\hline
\end{tabular}

Tabla 4. Probabilidad de ser menor o mayor de 18 años según sexo y estadio de Demirjian.

\section{DISCUSIÓN}

Al analizar la literatura se evidencio que existen varios métodos para evaluar la estimación de la edad, uno de los métodos más utilizados es el de Demirjian. El tercer molar es la única pieza que continúa su formación después de la adolescencia, la ausencia sea de tipo congénita o por exodoncias, las hacen no idóneas para ser utilizadas como marcador del desarrollo en edades tempranas. ${ }^{8,9}$ En varios estudios, que evalúan la estimación de la edad con el método Demirjian, las muestras presentan un mayor porcentaje del individuos del sexo femenino, mostrándose de igual manera que en el presente estudio, donde el sexo femenino formó 
parte del 58,50\% de la población evaluada. ${ }^{10,11}$

En un estudio realizado por Quezada y colaboradores en el año 2014, analizaron 1176 radiografías panorámicas digitales, donde al igual que este estudio los participantes tenían rangos de edad entre 7 a 23 años. Como resultado los autores encontraron que en la pieza dental 3.8 , el estadio D presentó una edad media de 14,05 \pm 1,35 años en las mujeres y de 13,42 $\pm 1,30$ años en los hombres, evidenciando que no existieron discrepancias estadísticamente significativas entre los estadios de Demirjian de las piezas 3.8 y 4.8. Por otra parte, los autores establecen que en el estadio $\mathrm{H}$ el $100 \%$ de mujeres y el 99,1\% de hombres tuvieron 18 o más años, algo similar sucede en el presente estudio, donde la pieza 3.8 en el estadio D presenta un promedio de edad muy parecido, siendo de 13,25 $\pm 2,17$ para el sexo femenino y de 13,07 $\pm 2,07$ para el sexo masculino; en cuanto al estadio $\mathrm{H}$ se constató que el $93,8 \%$ de las mujeres y el $96,1 \%$ de los hombres tuvieron 18 o más años. ${ }^{12}$

En el año 2016 Duangto et al. en su estudio aplicado en una población tailandesa, en individuos entre 8 y 23 años, evidenciaron que los hombres alcanzan etapas de formación de la raíz de terceros molares inferiores antes que las mujeres, obteniendo un alto coeficiente de correlación para los terceros molares mandibulares, tanto izquierdos como derechos, en ambos sexos $(r=0,945$ y $0,944, r=0,922$ y 0,923 en hombres y mujeres, respectivamente). Similar a nuestro estudio, donde el coeficiente de correlación para la pieza 3.8 en el sexo femenino fue de 0,8817 y en el sexo masculino de 0,915 , con respecto a la pieza 4.8 en el sexo femenino la correlación fue de 0,8844 y en el sexo masculino de $0,955 .{ }^{13}$

Venkatesh y Vishwanath en su estudio realizado en el año 2016, conjunto con el estudio realizado por Kasper y colaboradores, concluyeron que las etapas de desarrollo del tercer molar son útiles y fiables en la estimación de la edad dental, y no encontraron diferencias significativas entre los sexos para los cuatro terceros molares, evidenciando que todos los individuos que presentaban el tercer molar maduro tenían al menos 18 años de edad. ${ }^{10,14}$

En el año 2012, Li realizo un estudio en adolescentes chinos occidentales; donde examinó un total de 2078 radiografías panorámicas digitales, de 989 hombres y 1089 mujeres entre 5 y 23 años, observando que el desarrollo de terceros molares en la población china occidental era probable que comience a la edad de 5 años, resultados que difieren con el presente estudio, ya que el desarrollo de mineralización de la pieza 3.8 en las mujeres comienza a los 9,15 años $\pm 0,96$ (estadio A), y en los hombres a los 10,00 años $\pm 1,36$ (estadio A); para la pieza 4.8 el proceso de mineralización inicia a los 9,50 años $\pm 1,53$ (estadio A) en las mujeres, y a los 10,00 años $\pm 1,36$ (estadio A) en los hombres. ${ }^{11}$

Babburi et al., en su estudio desarrollado en India evaluaron un total de 550 radiografías panorámicas de individuos entre los 15 a 22 años de edad, donde obtuvieron como resultado que en el grupo de 15-22 años de edad no se evidencio estadios A y B de Demirjiam, a diferencia del presente estudio donde sí se reportó casos con estos estadios. ${ }^{5}$ Por otra parte, en el estudio publicado por Ajmal et al. en el año 2012, establecen que en el estadio $H$ se presentó una media de $22 \pm 1,77$ años, ${ }^{15}$ resultados similares a los de esta investigación, en la cual la muestra reveló un crecimiento radicular completo (estadio $\mathrm{H}$ ) de la pieza 3.8 a los 21,19 años en el sexo femenino) y a los 21,02 años en el sexo masculino, para la pieza 4.8 la media del estadio $\mathrm{H}$ fue a los 21,21 años en el sexo femenino y a los 21,01 en el sexo masculino.

A su vez en el año 2014 Naik y cols, en su estudio revelaron que el $88 \%$ de los sujetos mostró una correlación lineal estadísticamente significativa entre la edad cronológica y la edad de desarrollo utilizando el método de Demirjian, en nuestro estudio se evidencio un porcentaje de coincidencia del $88,92 \%^{16}$ El presente estudio al análisis estadístico mostró una fuerte correlación entre la edEl presente estudio al análisis estadístico mostró una fuerte correlación entre la edad y el desarrollo del tercer molar en ambos sexos, coincidiendo con lo encontrado por Rai en India, y por Heras en España, debido a que los autores sugieren que la etapa de Demirjian para el diente 3.8 puede considerarse un buen estimador de la edad. ${ }^{17,18}$ (Tabla 5)

\begin{tabular}{|c|c|c|c|c|c|c|c|c|c|}
\hline \multirow[t]{3}{*}{ Autores } & \multirow[t]{3}{*}{ Pais } & \multirow[t]{3}{*}{ Casos } & \multicolumn{2}{|c|}{ Sexo } & \multirow[t]{3}{*}{ Edad } & \multicolumn{4}{|c|}{$\begin{array}{l}\text { Probabilidad de ser mayoria de edad según } \\
\text { el estadio } \mathrm{H}\end{array}$} \\
\hline & & & \multirow[t]{2}{*}{ M } & & & \multicolumn{2}{|c|}{38} & \multicolumn{2}{|c|}{48} \\
\hline & & & & & & M & $F$ & M & \\
\hline Dominguez & Ecuador & 596 & $41,50 \%$ & $58,50 \%$ & $7-23$ & $96,08 \%$ & $93,75 \%$ & $96,00 \%$ & $93,80 \%$ \\
\hline Blankenshipi & EE. UU. & 1200 & $50,03 \%$ & $49,97 \%$ & $14-24$ & $89,90 \%$ & $93,40 \%$ & $89,90 \%$ & $93,40 \%$ \\
\hline Cantekin $^{20}$ & Turquia & 1348 & $46,10 \%$ & $53,85 \%$ & $7-22$ & - & - & - & - \\
\hline Quispe" & Perú & 208 & $49,04 \%$ & $50,96 \%$ & $14-22$ & $96,77 \%$ & $93,75 \%$ & $96,00 \%$ & $93,80 \%$ \\
\hline Duangto ${ }^{13}$ & Tallandia & 1867 & $46,97 \%$ & $53,02 \%$ & $8-23$ & $100 \%$ & $100 \%$ & $100 \%$ & $100 \%$ \\
\hline Venkatesh ${ }^{14}$ & India & 167 & $49,48 \%$ & $50,89 \%$ & $14-24$ & $96,59 \%$ & $96,59 \%$ & $96,59 \%$ & $96,59 \%$ \\
\hline Suărez $^{22}$ & Perú & 978 & $49,48 \%$ & $50,52 \%$ & $13-23$ & $89,08 \%$ & $86,49 \%$ & $86,21 \%$ & $85,54 \%$ \\
\hline Argüello ${ }^{23}$ & Perú & 402 & $46,51 \%$ & $53,49 \%$ & $14-22$ & $100 \%$ & $100 \%$ & $100 \%$ & $100 \%$ \\
\hline Babbur ${ }^{5}$ & India & 550 & $45,09 \%$ & $54,90 \%$ & $15-22$ & $90 \%$ & $90 \%$ & $90 \%$ & $90 \%$ \\
\hline Naik $^{16}$ & India & 100 & $53 \%$ & $47 \%$ & $7-24$ & - & - & - & - \\
\hline Quezada $^{12}$ & Perú & 1176 & $42,30 \%$ & $57,70 \%$ & $7-23$ & $99,20 \%$ & $100 \%$ & $99,10 \%$ & $100 \%$ \\
\hline Li11 & China & 2078 & $47,59 \%$ & $52,41 \%$ & $5-23$ & - & - & - & - \\
\hline de Oliveira 24 & Brasil & 407 & $49,38 \%$ & $50,61 \%$ & $6-25$ & $96,80 \%$ & $98,60 \%$ & $96,80 \%$ & $98,60 \%$ \\
\hline Verma ${ }^{28}$ & India & 220 & $50 \%$ & $50 \%$ & $14-24$ & - & - & - & - \\
\hline Kasper & EE. UU. & 950 & $44 \%$ & & $12-22$ & $85,80 \%$ & $89,90 \%$ & $86,40 \%$ & $87,10 \%$ \\
\hline Rail $^{18}$ & India & 250 & $49,60 \%$ & $50,40 \%$ & $7-26$ & - & - & - & - \\
\hline Mincer ${ }^{25}$ & EE. UU. & 823 & $54 \%$ & $46 \%$ & $14,1-24.9$ & $90,10 \%$ & $92,20 \%$ & $90,10 \%$ & $92,20 \%$ \\
\hline
\end{tabular}

Tabla 5. Resumen de estudios realizados en varias poblaciones.

En conclusión, a pesar que existe una fuerte correlación para ambos sexos entre los estadios de mineralización de la pieza 38 y 48 con la edad cronológica con el método de 
Demirjian no se puede determinar la edad exacta de una persona, pero si se puede descubrir la etapa de Demirjian en la que se encuentran. Como una posible explicación puede estar atribuida por su origen étnico, el tiempo o la diferencia entre los estudios sobre el desarrollo dental, lo cual hace evidente la necesidad de realizar un levantamiento de información aplicando esta metodología en cada población de América Latina.

\section{Conclusiones}

En conclusión, se corroboro una muy alta correlación entre la edad cronológica y los estadios de mineralización del tercer molar inferior en radiografías panorámicas digitales. Sin embargo, a pesar de que existe una fuerte correlación para ambos sexos, no se puede determinar la edad exacta de una persona, pero si se puede descubrir la etapa de Demirjian en la que se encuentran. Como una posible explicación puede estar atribuida, su origen étnico, el tiempo o la diferencia entre los estudios sobre el desarrollo dental, lo cual hace evidente la necesidad de realizar un levantamiento de información aplicando esta metodología en cada población de América Latina.

\section{Conflicto de Intereses}

Los autores declaran no tener conflicto de intereses.

\section{Referencias Bibliográficas}

1 Prieto J. La maduración del tercer molar y el diagnóstico de la edad. Evolución y estado actual de la cuestión. Cuad Med Forense. 2008; 14(51):11-24.

2 Demirjian A, Goldstein H, Tanner J. A new system of dental age assessment. Hum Bio. 1973; 45:211-27.

3 Darji J, Govekar G, Kalele S, Hariyani H. Age Estimation from Third Molar Development a Radiological Study. J Indian Acad Forensic Med. 2011; 33:130-134.

4 Espina Á, Fereira J, Céspedes M, Barrios F, Ortega A, Maldonado Y. Empleo de la edad dental y la edad ósea para el cálculo de la edad cronológica con fines forenses, en niños escolares con alteraciones en el estado nutricional, en Maracaibo, estado Zulia: Estudio preliminar. Acta Odontol Venez. 2007; 45(3):433-439.

5 Babburi S, Nelakurthi H, Aparna V, Soujanya P, Kotti A, Ganipineni K. Radiographic Estimation of Chronological Age using Mineralization of Third Molars in Coastal Andhra, India. Int Oral Health. 2015; 7(5):49-52.

6 Garamendi P, Landa M. Estimación forense de la edad en torno a 18 años: Revisión bibliográfica. Cuad. med. Forense. 2003; (31):13-24.

7 Bernaldo J, Caballero H. Método basado en el estudio del desarrollo de las terceras molares en la estimación de la edad cronológica [tesis de posgrado]. [Lima]: Facultad de Odontología, Universidad Nacional Mayor de San Marcos; 2017. 97 p.
8 Cadenas I, Celis C, Hidalgo A. Método de Demirjian para estimación de edad dentaria en base a estadios de mineralización. Anu. So c. Radiol. 2010; 13:17-23.

9 Prieto J, Abenza J. Métodos para valorar la edad en el adolescente. Rev. Esp. Med. Leg.1998; (84-85):45-50.

10 Kasper K, Austin D, Kvanli A, Rios T, Senn D. Reability of third molar development for age estimation in a Texas Hispanic population: a comparation study. J Forensic Sci. 2009; 54(3):651-657.

11 Li G, Ren J, Zhao S, Liu Y, Li N, Wu W. Dental age estimation from the developmental stage of the third molars in western Chinese population. Forensic Science International. 2012; 219:158-164.

12 Quezada M, Beltran J, Bernal J, Evangelista A, del Castillo C. Relación entre la edad cronológica y la mineralización del tercer molar inferior según método de Demirjian. Rev Estomatol Herediana. 2014; 24(2):63-72.

3 Duangto P, Iamaroon A, Prasitwattanaseree S, Mahakkanukrauh P, Janhom A. New models for age estimation and assessment of their accuracy using developing mandibular third molar teeth in a Thai population. J Legal Med. 2017; 131(2):559-568.

14 Venkatesh M, Vishwanath S. The chronology of third molar mineralization by digital Orthopantomography. Journal of Forensic and Legal Medicine. 2016; 43:70-75.

15 Ajmal M, Assiri K, Al-Ameer K, Assiri A, Luqman M. Age estimation using third molar teeth: A study on southern Saudi population. J Forensic Dent Sci. 2012; 4(2):63-5.

16 Naik S, Patil S, Kamble S, Mowade T, Motghare P. Reliability of third molar development for age estimation by radiographic examination (Demirjian's method). J. Clin. Diagn. Res. 2014; 8(5): ZC25-ZC28.

17 Heras M, García P, Ortega A, Zodocovich S, Valenzuela A. Third molar development according to chronological age in populations from Spanish and Magrebian origin. Forensic Sci Int. 2008;174(1):47-53.

18 Rai B, Krishan K, Kaur J, Anand SC. Technical note: Age estimation from mandible by lateral cephalogram: a preliminary study. J Forensic Odontostomatol. 2008; 26(1):24-8.

19 Blankenship J, Mincer H, Anderson K, Woods M, Burton E. Third molar development in the estimation of chronologic age in american blacks as compared with whites. J Forensic Sci. 2007; 52(2):428-33.

20 Cantekin K, Yilmaz Y, Demirci T, Celikoglu M. Morphologic analysis of third-molar mineralization for eastern Turkish children and youth. J Forensic Sci. 2012; 57(2):531534.

21 Quispe L, Solís A, Quezada M, Cameriere R. Demirjian's stages and Cameriere's third molar maturity index to estimate legal adult age in Peruvian population. Leg Med. 2017; 25:59-65.

22 Suárez C. Eficacia de los métodos utilizados para estimar la edad de personas de 13 a 23 años [tesis de posgrado]. [Lima]: Facultad de Odontología, Universidad Nacional 
Mayor de San Marcos; 2016. 172 p.

23 Argüello J. Validación de la estimación de la edad cronológica mediante del estudio radiológico de las terceras 26 molares usando el método de Demirjian [tesis de posgrado]. [Lima]: Facultad de Odontología, Universidad Nacional Mayor de San Marcos; 2015. 172 p. 111.

24 de Oliveira F, Capelozza A, Lauris J, de Bullen I. Mineralization of mandibular third molars can estimate chronological age Brazilian indices. Forensic Sci Int. 2012; 219(10):147-150.

25 Verma P, Sachdeo J, Verma K, Gupta S, Guruprasad R. Age estimation of adolescents and young adults based on deve- lopment of mandibular third molars: A panoramic study. J Indian Acad Oral Med Rad. 2011; 23(1):9-13.

6 Mincer H, Harris E, Berryman H. The A.B.F.O. study of third molar development and its use as an estimator of chronological age. J Forensic Sci. 1993; 38(2):379-390.

Recibido: 12 de Abril de 2020

Aceptado: 20 Junio de 2020 